\title{
Anonymous Giving as a Vice: An Application of Image Motivation
}

\author{
Masaoki Tamura \\ Graduate School of Economics, University of Tokyo, Tokyo, Japan \\ Email: tamuramasaoki@gmail.com
}

Received June 6, 2012; revised July 2, 2012; accepted August 1, 2012

\begin{abstract}
While some donors boast about their giving, others give anonymously. A novel feature of this paper is that anonymity is endogenously controlled by donors themselves, not exogenously controlled by fundraisers. Is anonymous giving really a virtue, as is generally recognised? Paradoxically, this paper proves that anonymity is a vice for fundraisers even if it is what donors desire. If many altruists (a good type) switch from known to anonymous giving, it relatively lowers the group reputation of known donors and enhances that of non-donors. These effects dilute the incentive for other individuals to become known donors, if they have psychological "image motivation". I suggest a practical method to control the expected number of anonymous donors: fundraisers remove the "check boxes" from their web sites.
\end{abstract}

Keywords: Giving; Anonymity; Altruism; Image; Fundraising

\section{Introduction}

Many studies, including [1-3], show that if experimenters control the anonymity of examinees, the examinees change their prosocial behaviour. The more anonymity experimenters provide, the less altruistic the examinees become. A novel feature of this paper is that anonymity is endogenously determined by the donors themselves. While some donors boast about their giving, others give anonymously. Is anonymous giving worth the same as known giving to fundraisers? Is anonymous giving really a virtue, as is generally recognised? Paradoxically, this paper proves that anonymous giving is a vice, even if the donors themselves desire anonymity.

We extend Benabou and Tirole's image signalling framework to the case in which "anonymity" is redefined and individuals themselves choose anonymity level. Since people care about their social reputations, even non-altruists donate to conceal their selfishness and enhance their reputations. The key lies in this "hypocritical" behaviour of donors. Such behaviour is possible because altruism is private information and the donation amount is what others observe; giving enables non-altruists to mimic altruists and achieve recognition as altruists. However, if we allow donors an option to anonymous giving, it is clear that not all donors choose the same anonymity level. It is essential to identify who donates anonymously, because it determines whether anonymous giving is independent of or related to the hypocritical behaviour. Our result is that those who prefer anonymous giving are the best "target" for the non-altruists to mimic, and the exis- tence of anonymous donors negatively affects the hypocritical behaviour of non-altruists and the total amount of donation.

Practically, fundraisers should control the expected number of anonymous donors. However, we can find one example that exaggerates the existence of anonymous donors. Currently, many fundraisers solicit contributions via the Internet in addition to normal fundraising activities. On their Web sites, some fundraisers place check boxes for donors to select anonymous or known donations. Figure 1 shows how a typical such site looks. On the page, donors fill out not only the information about the amount of donation, name, address, and credit card number but also the check box "I prefer to make this donation anonymously". By providing the check boxes, these fundraisers intend to make it convenient to donate anonymously. The problem, however, is that, when facing the choice, donors expect that fundraisers provided the check box in response to many requests from a substantial number of anonymous donors. In other words, the check box exaggerates the number of anonymous donors. The contribution of this paper is threefold. First and foremost, it suggests how fundraising campaigns can be more efficiently designed: fundraisers should allow anonymous donation only as an exception and should not exaggerate the number of anonymous donors. Specifically, we can identify inefficiency in some fundraising Web sites and suggest an improvement. Such sites can impose a small "foot cost" on anonymous giving. Second, this is the first paper that studies a model in which anonymity is 


\section{Donate Online}

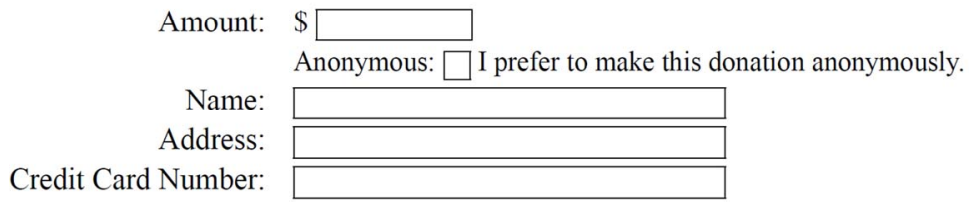

Figure 1. Online donation page.

endogenously determined. Here, anonymity is controlled not by the fundraisers but by the donors themselves. Thus, we can study the case in which donors choose perfect anonymity, namely by donating anonymously; almost no economic studies have addressed this case in detail. Third, the study gives weight to the heterogeneity of image motivation among individuals, in contrast to [4]. Thus, we can identify who donates anonymously

\section{Structure of the Model}

Let us begin our analysis by specifying the utility function of the individuals who have both an image motivation and a warm-glow preference ${ }^{1}$.

$$
U\left(c_{i}, x_{i}, \hat{\alpha}_{i}\right)=\left(1-\alpha_{i}\right) \ln c_{i}+\alpha_{i} \ln x_{i}+\beta_{i} \ln I\left(\hat{\alpha}_{i}\right)
$$

$c_{i}$ denotes individual $i$ 's consumption for private goods, $x_{i}$ denotes individual i's donation, $\alpha_{i}$ denotes the altruism parameter, and $\beta_{i}$ denotes the self-consciousness of consumer $i$. Individuals are heterogeneous in the parameters $\alpha_{i}$ and $\beta_{i}$. Because we assume that individual i's parameters $\alpha_{i}$ and $\beta_{i}$ are private information and unobservable to all other individuals, others form beliefs about $i$ 's parameters. $\hat{\alpha}_{i}$ denotes the belief of other individuals about i's altruism, $\alpha_{i}$. Individuals derive utility directly from this belief of others, namely, the extent to which others consider an individual altruistic. This corresponds to the concept of self-image or image motivation. The function $I(\cdot)$ represents this image motivation of the individuals. We assume that $I^{\prime}(\cdot)>0$ and that $I(0)=1$. People who are considered socially altruistic have a high $\hat{\alpha}_{i}$ and hence high utility. The more altruistic they are considered the more utility they gain. Here, the heterogeneity lies in altruism $\alpha_{i}$ and self-consciousness beta $a_{i}$, which we specify below.

The budget constraint for $i$ is

$$
\begin{gathered}
c_{i}+x_{i}=y_{i}, \\
x_{i}=D_{i} x_{k i}+\left(1-D_{i}\right) x_{a i} .
\end{gathered}
$$

$y_{i}$ is the endowment of individual $i$. People allocate their income between consumption and donation. $x_{k}$ is known donation, $x_{a}$ is anonymous donation, and $D_{i}$

${ }^{1}$ Theoretical and empirical backgrounds for these two motivations are given in [4-10]. is a dummy variable that takes the value 1 if individual $i$ chooses known over anonymous donation, and takes the value 0 if individual $i$ chooses anonymous over known donation. In short, in this model, when individuals want to donate, they must choose between a known and an anonymous donation. For simplicity, we assume that individuals cannot make both anonymous and known donations at the same time. We must draw attention to the implied assumption that the choice between anonymous and known donation does not directly affect the utility: both choices yield the same warm-glow utility. However, the choice does matter when individuals form beliefs about others' $\alpha$ values: $\hat{\alpha}_{i} \cdot \hat{\alpha}_{i}$ is formed by

$$
\hat{\alpha}_{i}=E\left[\alpha_{i} \mid x_{k i}, y_{i}\right] .
$$

The beliefs about others' altruism are formed based on income and known donation. In other words, individual's decision variables except known donation are unobservable to the others. It must be noted that individuals form beliefs about $\alpha_{i}$ based on $x_{i k}$, not $x_{i}$. This result is owing to the definition of anonymous donation: anonymous donation is unobservable to others, while known donation is observable. It is important that anonymous donors $\left(x_{a} \neq 0\right.$ and $\left.x_{k}=0\right)$ and non-donors $\left(x_{a}=0\right.$ and $x_{k}=0$ ) are considered the same by others.

Next, we specify the parameter values and the distribution of the individual types in this economy. Table 1 summarises the information about the four individual types of our model.

\section{The Equilibrium}

\subsection{Who Gives Anonymously?}

The first task is to determine who donates anonymously. The answer is the "pure altruists". There are two reasons for this. First, because anonymous donation, by definition, cannot be observed by others, anonymous donors and non-donors are considered the same (i.e. the same $\hat{\alpha}_{i}$ ). Second, the types who are concerned with their own reputation ( $\beta \neq 0$ ) want to be seen as altruistic (i.e., as donors); consequently, they never prefer anonymous to known donation. Then, the "pure altruists" (who are altruistic but not self-conscious) is the only type who may make anonymous donations. Pure altruists must be 
Table 1. Individual Types.

\begin{tabular}{cccc}
\hline Type & $\alpha$ & $\beta$ & Population \\
\hline Selfish & 0 & 0 & $N_{s}$ \\
Pure Altruist & $\bar{\alpha}$ & 0 & $N_{p a}$ \\
Hypocrite & 0 & $\bar{\beta}$ & $N_{h}$ \\
Impure Altruist & $\bar{\alpha}$ & $\bar{\beta}$ & $N_{i a}$ \\
\hline
\end{tabular}

indifferent to whether a donation is known or anonymous.

\subsection{Equilibrium Actions}

From now on, we examine the behaviour of each type to find the Nash equilibrium of our model. It should be noted that individuals interact only through the image $\left(\hat{\alpha}_{i}\right)$ of the types to which they belong. Thus, people without self-consciousness (i.e., $\beta_{i}=0$ ) behave without considering others' actions. We first limit our attention to these types: the selfish and the pure altruists.

\subsubsection{Selfish: $\alpha=0 \quad \& \quad \beta=0$}

People without self-consciousness, by definition, simply solve their classical optimisation problems with respect to donation and private good, but not their images. In short, it is the simple warm-glow setup we see above. It is obvious that the selfish spend all of their income on private goods. For the selfish, $c_{i}=y_{i}$ and $x_{i}=0$.

\subsubsection{Pure Altruist: $\alpha=\bar{\alpha} \quad \& \quad \beta=0$}

Pure altruists also face their classical optimisation problems without image motivation. The solution to this problem is $c_{i}=(1-\bar{\alpha}) y_{i}$ and $x_{i}=\bar{\alpha} y_{i}$. Because $\hat{\alpha}_{i}$ does not appear in their optimisation problems, pure altruists are indifferent to the choice between $x_{a}$ and $x_{k}$; both options yield the same utility. Here, we assume that a fraction $A \in(0,1)$ of the pure altruists choose anonymous over known donation, and thus, $1-A$ of them choose known over anonymous donation. As a result, the introduction of the option of anonymous giving makes some pure altruists switch from known to anonymous giving. We should not overlook that the total donation amount of pure altruists is independent of $A$.

\subsubsection{Hypocrite: $\alpha=0 \quad \& \quad \beta=\bar{\beta}$}

It is most important to examine the conditions under which even hypocrites make donations in spite of their selfish nature. By making donations, they mimic the pure altruists (a good type) to enhance their reputations. Note that the pure altruists are the best "target" for the hypocrites to mimic, because the impure altruists are averse to mimicry as is described later.
It can be checked easily that there are only two options for hypocrites to choose, $x_{i}=0$ or $x_{i}=\bar{\alpha} y_{i}$. If they choose $x=0$, then they are in the same group as the selfish. Conversely, if they choose $x=\bar{\alpha} y_{i}$, then they are in the same group as the pure altruists. Comparing the two options, the condition for hypocrites to choose $x=\bar{\alpha} y_{i}$ over $x=0$ is ${ }^{2}$

$$
\begin{aligned}
& \ln \left(y_{i}-\bar{\alpha} y_{i}\right)+\bar{\beta} \ln I\left(\frac{(1-A) N_{p a}}{N_{h}+(1-A) N_{p a}} \bar{\alpha}\right) \\
> & \ln y_{i}+\bar{\beta} \ln I\left(\frac{A N_{p a}}{N_{h}+A N_{p a}+N_{s}} \bar{\alpha}\right) .
\end{aligned}
$$

The left hand side represents the utility when hypocrites join the group of known donors $\left(x_{i}=\bar{\alpha} y_{i}\right)$, while the right hand side represents the utility when they join the group of non-donors $\left(x_{i}=0\right)$. In conclusion, the more anonymous donors there are, the less likely hypocrites are to donate. Let us mathematically confirm this result and explain the intuition behind it. The second term on the left hand side of (5) is decreasing in $A$,

$$
\frac{\partial \bar{\beta} \ln I\left(\frac{(1-A) N_{p a}}{N_{h}+(1-A) N_{p a}} \bar{\alpha}\right)}{\partial A}<0,
$$

and the right hand side of (5) is increasing in $A$,

$$
\frac{\partial \bar{\beta} \ln I\left(\frac{A N_{p a}}{N_{h}+A N_{p a}+N_{s}} \bar{\alpha}\right)}{\partial A}>0 .
$$

The interpretation of these two inequalities is the core of this paper. We refer to (6) as the "decrease effect", and (7) as the "blend effect". We first note that pure altruists are thought to be a "good" type compared with the selfish and the hypocrites because pure altruists have higher altruism. Next, the group reputation is formed according to the ratio of "good" (altruistic) group members. To interpret (6), suppose that some of the pure altruists (a "good" type) switch from known $\left(\left(x_{a}, x_{k}\right)=\left(0, \bar{\alpha} y_{i}\right)\right)$ to anonymous donation $\left(\left(x_{a}, x_{k}\right)=\left(\bar{\alpha} y_{i}, 0\right)\right)$. Such a switch implies a decrease of a "good' type in the group of known donors $\left(x_{k}=\bar{\alpha} y_{i}\right)$. Then, to the hypocrites, joining the group of known donors $\left(x_{k}=\bar{\alpha} y_{i}\right)$ becomes less attractive. This decrease effect corresponds to inequality (6). The second inequality (7) represents the blend effect. Because anonymous donation is unobservable, people now think that some perceived non-donors are actually anonymous donors. Some fraction of non-donors is of the "good" type (

\footnotetext{
${ }^{2}$ For simplicity, we consider the case in which all hypocrites behave as a group. This does not change the result much as long as we focus on the symmetric equilibrium.
} 


$$
\hat{\alpha}=\frac{A N_{p a}}{N_{h}+A N_{p a}+N_{s}} \bar{\alpha}>0
$$

on the left hand side of (7)). As a result, the existence of anonymous donors enhances the reputation of non-donors, and joining the group of non-donors $\left(x_{k}=0\right)$ becomes more attractive to the hypocrites. Here, anonymous donors blend into non-donors. This blend effect corresponds to inequality (7).

A numerical simulation is shown in Figure 2, "Utility of Hypocrites"3. The "Join Donors" curve corresponds to the left hand side of (5) for each $A$. The "Join Non-Donors" curve corresponds to the right hand side of (5) for each $A$. We refer to the value of $A$ for which the left hand side equals the right hand side as $A^{*}$. If there are anonymous donors above $A^{*}=17.7 \%$, then hypocrites do not donate.

\subsubsection{Impure Altruist: $\alpha=\bar{\alpha} \quad \& \quad \beta=\bar{\beta}$}

If hypocrites mimic pure altruists, the reputations of the hypocrites improve, as is described above. In contrast, the reputations of the pure altruists worsen because they are now in the same group as hypocrites (a "bad type"). Even then, the equilibrium action of pure altruists is unchanged because they do not derive utility from the reputation (i.e., $\beta=0$.)

Contrary to pure altruists, impure altruists care about their own reputation (i.e., $\beta=\bar{\beta} \neq 0$ ). Impure altruists are averse to be mimicry by hypocrites. First, when $A$ is below $A^{*}$, hypocrites mimic pure altruists. If $A$ is increasing from 0 , then pure altruists become increasingly less attractive to mimic than impure altruists. In response,
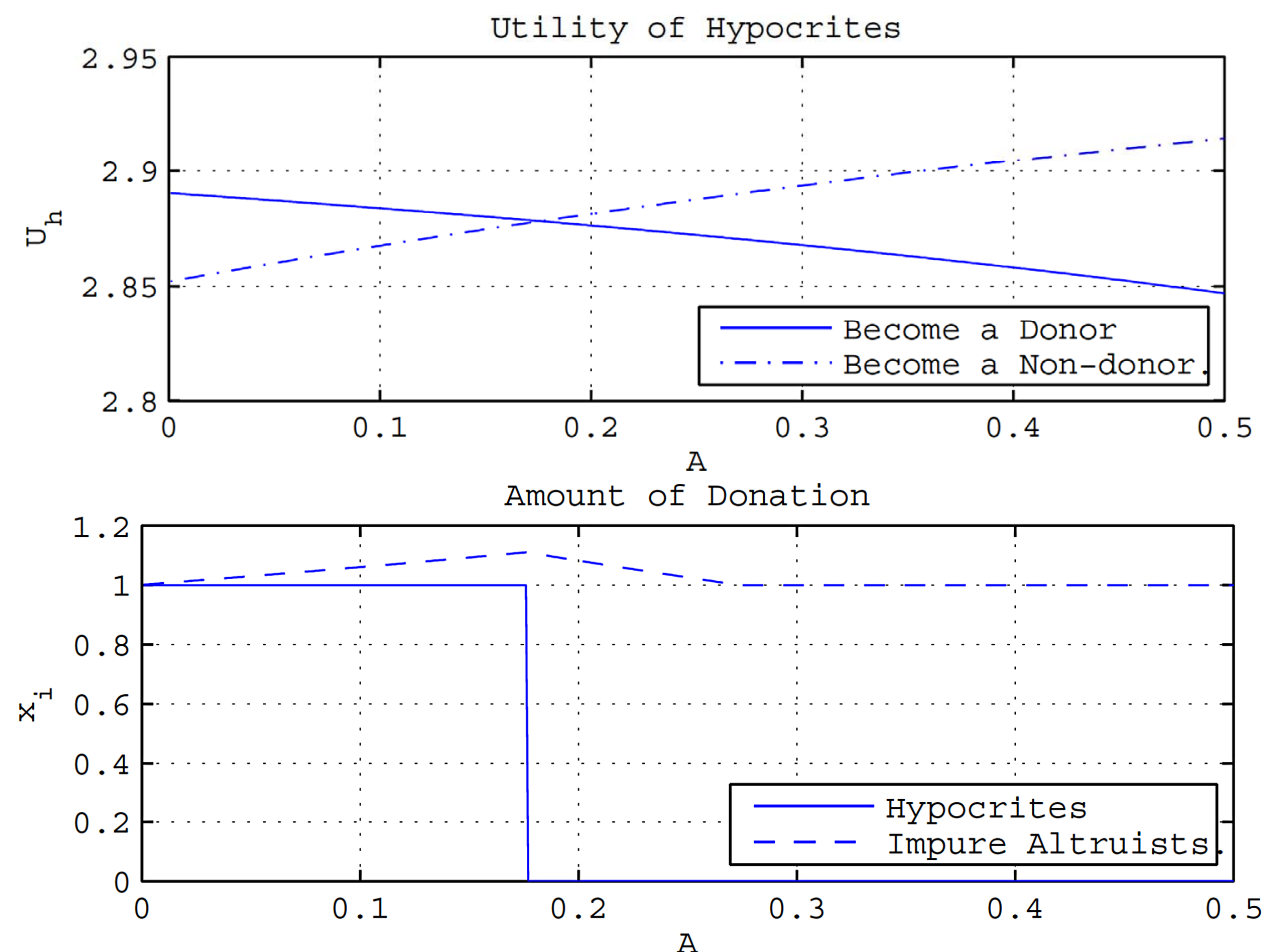

Total Amount of Donation

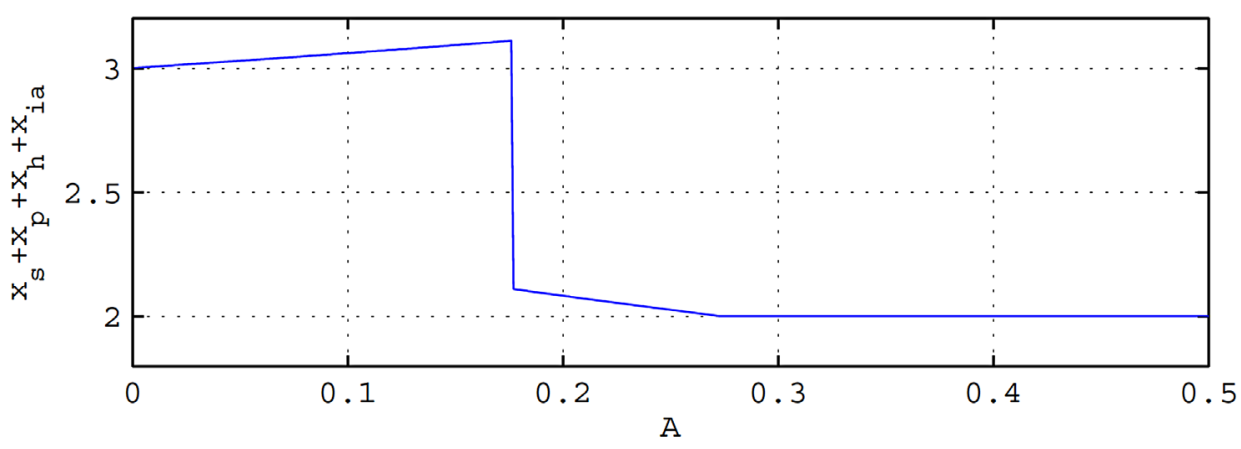

Figure 2. Numerical simulation.

${ }^{3}$ The values used in our numerical simulations are $\bar{\alpha}=0.1, \bar{\beta}=0.5, y_{i}=10 \quad \forall i$, and $I\left(\hat{\alpha}_{i}\right)=3+20 \hat{\alpha}_{i}$. 
impure altruists want to become less attractive because they are averse to mimicry by hypocrites. Then, impure altruists gradually increase their donations as $A$ becomes large because larger-amount donors are less attractive for hypocrites to mimic. Secondly, when $A$ surpasses $A^{*}$, hypocrites are non-donors. If $A$ is increasing from $A^{*}$, for hypocrites, being non-donors yields increasing utility compared with mimicking impure altruists. In response, impure altruists can decrease their donation amounts to their original ideal level, $x_{k}=\bar{\alpha} y_{i}$.

\subsection{Total Amount of Donation}

While impure altruists are averse to be mimicry by hypocrites, pure altruists do not care about it. Thus, the hypocrites mimic the pure altruists. The existence of anonymous donors means 1) the decrease of the best "target" for the hypocrites to mimic and 2) the increase of the "good" type in the non-donor group. The total amount of donation of these four types is shown in Figure 2. In this numerical example, $A^{*}=0.177$. The sharp decrease at $A^{*}=0.177$ is due to the hypocrites' behaviour: they no longer mimic pure altruists. In conclusion, for fundraisers to raise as much money as possible, it is crucial that $A$ does not exceed $A^{*}$, though a small fraction of anonymous donors is not harmful.

\section{Policy Implication and Discussions}

In the preceding section, we examine how the total donation amount varies according to an exogenously determined $A$. This result raises the question, can fundraisers control $A$ and expected $A$ ? In this section, we discuss one practical method to control both $A$ and expected A.

Currently, many fundraisers solicit contributions via the Internet in addition to normal fundraising activities. On their Web sites, some fundraisers including Anna Marie's Alliance, the Minnesota Aids Project, and Network for Good place check boxes for donors to select either anonymous or known donations. Figure 1 shows how a typical such site looks. Donors fill out not only information about the donation amount, name, address, and credit card number but also a check box, "I prefer to make this donation anonymously”. However, some organisations, such as the American Cancer Society, the American Red Cross, and Doctors Without Borders do not provide such a check box on their Web sites. Without a special request, only known donation is available for donors. We see that not a few fundraisers explicitly offer opportunities for anonymous giving. By providing the check boxes, these fundraisers intend to make it convenient to donate anonymously. The problem, however, is that, when facing the choice, donors expect that fund- raisers provided the check box in response to many requests from a substantial number of anonymous donors. In other words, the check box exaggerates the number of anonymous donors. Here, $A$ is expected higher than that it actually is.

To keep both $A$ and expected $A$ below $A^{*}$, fundraisers can remove this type of check box. Instead, they can implement some type of small foot cost on anonymous giving and accept anonymous donations only as an exception. For instance, to give anonymously, donors have to send an e-mail to fundraisers in addition to filling out the personal information form. Then, those who require anonymity choose anonymous giving with a small effort, while those who are indifferent to whether the donation is anonymous choose known giving.

An important experimental finding is reported in [1]. They show that, if examinees give subjects an option to donate anonymously, subjects increase their giving. Note that the subjects mainly increase known giving, not anonymous giving. Their finding is not inconsistent with our theoretical results, because their finding corresponds to the case of $A<A^{*}$ in our model. Our model predicts that if $A$ is small, impure altruists increase known giving above their ideal amount. This is what [1] observes. We also predict that if we would make $A$ and expected A sufficiently large, then the subjects would decrease their known giving. Fundraisers should keep the actual $A$ and expected $A$ at a low level and consequently maximise the total donation amount.

\section{Acknowledgements}

I would like to thank Masayuki Otaki for his helpful comments. I am also grateful to Sakuya Tamura for her support and fruitful discussions.

\section{REFERENCES}

[1] J. Andreoni and R. Petrie, "Public Goods Experiments without Confidentiality: A Glimpse into Fund-Raising," Journal of Public Economics, Vol. 88, No. 7-8, 2004, pp. 1605-1623. doi:10.1016/S0047-2727(03)00040-9

[2] M. Rege and K. Telle, "The Impact of Social Approval and Framing on Cooperation on Public Good Situations," Journal of Public Economics, Vol. 88, No. 7-8, 2004, pp. 1625-1644. doi:10.1016/S0047-2727(03)00021-5

[3] A. R. Soetevent, "Anonymity in Giving in a Natural Context-A Field Experiment in 30 Churches," Journal of Public Economics, Vol. 89, No. 11-12, 2005, pp. 23012323. doi:10.1016/j.jpubeco.2004.11.002

[4] R. Benabou and J. Tirole, "Incentives and Prosocial Behavior,” The American Economic Review, Vol. 96, No. 5, 2006, pp. 1652-1678.

[5] D. Ariely, A. Bracha and S. Meier, "Doing Good or Doing Well? Image Motivation and Monetary Incentives in Behaving Prosocially," The American Economic Review, 
Vol. 99, No. 1, 2009, pp. 544-555.

doi:10.1257/aer.99.1.544

[6] J. Carpenter and C. K. Myers, "Why Volunteer? Evidence on the Role of Altruism, Image, and Incentives,” Journal of Public Economics, Vol. 94, No. 11-12, 2010, pp. 911920. doi:10.1016/j.jpubeco.2010.07.007

[7] J. Andreoni, “Giving with Impure Altruism: Applications to Charity and Ricardian Equivalence,” Journal of Political Economy, Vol. 97, No. 6, 1989, pp. 1447-58. doi:10.1086/261662

[8] J. Andreoni, "Impure Altruism and Donations to Public
Goods: A Theory of Warm-Glow Giving,” The Economic Journal, Vol. 100, No. 401, 1990, pp. 464-477.

doi:10.2307/2234133

[9] H. Crumpler and P. J. Grossman, "An Experimental Test of Warm Glow Giving,” Journal of Public Economics, Vol. 92, No. 5-6, 2008, pp. 1011-1021.

doi:10.1016/j.jpubeco.2007.12.014

[10] T. R. Palfrey and J. E. Prisbrey, "Anomalous Behavior in Public Goods Experiments: How Much and Why?” The American Economic Review, Vol. 87, No. 5, 1997, pp. 829846. 